\title{
On hyperbolic systems with entropy velocity covariant under the action of a group
}

\author{
François Dubois ${ }^{a, b}$ \\ ${ }^{a}$ Conservatoire National des Arts et Métiers, \\ Laboratoire de Mécanique des Structures et des Systèmes Couplés, Paris, France. \\ ${ }^{b}$ Department of Mathematics, University Paris-Sud, \\ Bât. 425, F-91405 Orsay Cedex, France \\ francois.dubois@math.u-psud.fr
}

31 august $2014{ }^{1}$

\begin{abstract}
For hyperbolic systems of conservation laws in one space dimension with a mathematical entropy, we define the notion of entropy velocity. Then we give sufficient conditions for such a system to be covariant under the action of a group of space-time transformations. These conditions naturally introduce a representation of the group in the space of states. We construct such hyperbolic system from the knowledge of data on the manifold of null velocity. We apply these ideas for Galileo, Lorentz and circular groups. We focus on particular non trivial examples for two by two systems of conservation laws.
\end{abstract}

Mots-clés : Galileo group, Lorentz group, circular group, mathematical entropy.

AMS classification: 35L40, 58J45.

1 This contribution in honor of Jean-Marie Souriau (1922 - 2012) is issued from two lectures entitled "Sur les systèmes hyperboliques à vitesse entropique invariants sous l'action d'un groupe" given on tuesday 28 august 2012 and "On hyperbolic systems with entropy velocity covariant under the action of a group" given on wednesday 21 august 2013 at the 56th and 57th "Colloque International de Théories Variationnelles", La Baume, Aix en Provence, France. Published in the Journal of Hyperbolic Differential Equations, volume 12, number 4, pages 763-785, 2015, doi: 10.1142/S0219891615500228. Edition 23 january 2016. 


\section{FranÇOIS DUBOIS}

\section{1) Introduction}

- The first link between covariance under the action of a group and conservation laws is the classic result of Emmy Noether [16]: when the action is invariant under the action of a group, an associated moment is conserved along the time evolution. This result is well understood for systems that possess some action. This result is also fundamental for all the modern physics. It has been intensively studied and generalized by Jean-Marie Souriau in his fundamental book [19] (see also the work of Kostant [11]). The fact to consider Galilean relativity is natural in this kind of work. In order to explicit the mass as an invariant, the Bargmann group [1] is necessary in this framework and we refer to the work of de Saxcé and Vallée [4 for the introduction of the second principle of thermodynamics. Observe also that very early Souriau has considered not only Galilean relativity but also Einstein's relativity [18, 20, 21]. An other contribution is due to Vallée [22 relative to continuum media in the framework of special relativity.

- Our scientific background is first concerned with the approximation of gas dynamics problems with numerical methods [5]. The notion of first order hyperbolic system of conservation laws is fundamental for this study and the contribution of Peter lax (see e.g. [13]) is essential for all that follows. The introduction of the second principle of thermodynamics in this framework is due to Godunov [10] and to Friedrichs and Lax [8] with the notion of "mathematical entropy". A fundamental result of existence and uniqueness of an entropy solution in the scalar case is due to Kružkov [12]. Galilean conservation for hyperbolic systems has been proposed by Ruggeri [17]. We have considered the same question with enforcing the structure of the mathematical entropy in [6]. We consider an extension of this approach in this contribution.

- The summary of our study is the following. We introduce the entropy velocity at Section 2 for hyperbolic systems with a mathematical entropy. Then we define in Section 3 the notion of covariance for an hyperbolic system with entropy velocity under the action of a group and propose sufficient conditions that make in evidence an algebraic structure. The manifold of null velocity defined at Section 4 is a natural notion in ths context. Then precise constraints for the entropy variables are derived at Section 5. In Section 6, we study the particular case of systems of order two. Some words of conclusion are proposed at Section 7.

\section{2) Entropy velocity}

- Let $N$ be a positive integer and $\Omega$ a non void convex open part of $\mathbb{R}^{N}$. The physical flux $f(W) \in \mathbb{R}^{N}$ of a state $W \in \Omega$ is a regular function $f: \Omega \longrightarrow \mathbb{R}^{N}$. We study in the following the system of conservation laws in one space dimension with unknown $\mathbb{R} \times[0,+\infty[\ni(x, t) \longmapsto W(x, t) \in \Omega$ associated with this nonlinear flux function:

$$
\frac{\partial W}{\partial t}+\frac{\partial}{\partial x} f(W)=0
$$

- As proposed by Godunov [10], Friedrichs and Lax [8] and Boillat [2], a mathematical entropy for the system (1) is a regular strictly convex function $\eta: \Omega \longrightarrow \mathbb{R}$ such that for 
any regular solution $W(x, t)$ of the conservation law (1), there exists a complementary conservation equation

$$
\frac{\partial \eta}{\partial t}+\frac{\partial \zeta}{\partial x}=0
$$

The space derivative of the second term in 2 is the entropy flux, a regular function $\zeta: \Omega \longrightarrow \mathbb{R}$ that satisfies the identity

$$
\mathrm{d} \zeta \equiv \mathrm{d} \eta \bullet \mathrm{d} f .
$$

It is well known (see e.g. Godlewski - Raviart [9] or [5]) that a system of conservation law which admits a mathematical entropy is hyperbolic: the jacobian $\mathrm{d} f(W)$ is diagonalisable in $\mathbb{R}^{N}$. Observe also that the restriction to only one space dimension is not restrictive in principle if we adopt a point of view founded on mathematical entropy. The generalization to several space dimensions does not add in priciple new fundamental difficulties. As well known, the equality (2) has to be replaced by an inequality (taken in an appropriate weak sense) when discontinuous solutions of the conservation law (1) are considered. The co-vector $\varphi$ of entropy variables is the jacobian of the entropy relatively to the variation of the state $W$ :

$$
\mathrm{d} \eta \equiv \varphi \cdot \mathrm{d} W, \quad \forall W \in \Omega .
$$

The dual entropy $\eta^{*}$ is the Legendre [14] - Fenchel [7] - Moreau [15] transform of the convex function $\eta$. It is a function of the entropy variables:

$$
\eta^{*}(\varphi) \equiv \sup _{W \in \Omega}(\varphi \cdot W-\eta(W))
$$

and we have

$$
\mathrm{d} \eta^{*}(\varphi)=\mathrm{d} \varphi \cdot W
$$

- In the following, we define the "entropy velocity" $u$ as the quotient of the entropy flux divided by the entropy. In other terms, we suppose that the system (1) (2) admits an entropy velocity $u$ if there exists a regular function $u: \Omega \longrightarrow \mathbb{R}$ such that the entropy flux $\zeta$ can be written under the form $\eta u$ :

$$
\zeta(W) \equiv \eta(W) u(W), \quad \forall W \in \Omega .
$$

This definition has been previously proposed in [6]. For gas dynamics this entropy velocity is the usual velocity because the specific entropy is advected by the flow (see e.g. [5] or [9]). Once the velocity is defined, it is natural, as suggested by Ruggeri [17], to decompose the physical flux $f$ into a convective part $u W$ and a complementary contribution $g$. We define the "thermodynamic flux" $g(W)$ by the relation

$$
f(W) \equiv u(W) W+g(W), \quad \forall W \in \Omega .
$$




\section{FranÇOIS DUBOIS}

- Proposition 1. Compatibility relation

With the above framework, the compatibility relation (3) is satisfied if and only if

$$
\varphi \cdot \mathrm{d} g+\eta^{*} \mathrm{~d} u \equiv 0 \text {. }
$$

\section{Proof of Proposition 1.}

The proof has been explicited in [6]; we detail it for completeness. We have, due to the relations (3) to (8), the following calculus:

$$
\begin{gathered}
\mathrm{d} \zeta-\mathrm{d} \eta \bullet \mathrm{d} f=\mathrm{d}(\eta u)-\mathrm{d} \eta \bullet \mathrm{d}(u W+g)=\eta \mathrm{d} u+u \mathrm{~d} \eta-\varphi \bullet[(\mathrm{d} u) W+u \mathrm{~d} W+\mathrm{d} g] \\
\quad=(\eta-\varphi \bullet W) \mathrm{d} u+u(\mathrm{~d} \eta-\varphi \bullet \mathrm{d} W)-\varphi \bullet \mathrm{d} g=-\eta^{*} \mathrm{~d} u-\varphi \bullet \mathrm{d} g
\end{gathered}
$$

and the conclusion is clear.

\section{3) Covariance under the action of a group}

- We introduce a group $\mathcal{G}$ with one real parameter $\theta$ composed by transformations $G_{\theta}$ of space-time defined by a two by two matrix:

$$
G_{\theta}=\left(\begin{array}{cc}
\alpha_{\theta} & \delta_{\theta} \\
\beta_{\theta} & \gamma_{\theta}
\end{array}\right), \quad G_{\theta} \in \mathcal{G}, \quad \theta \in \mathbb{R} .
$$

At a fixed $\theta$, the transformation $(x, t) \longmapsto\left(x^{\prime}, t^{\prime}\right)$ is defined by the relations:

$$
\left(\begin{array}{l}
x^{\prime} \\
t^{\prime}
\end{array}\right)=G_{\theta}\left(\begin{array}{l}
x \\
t
\end{array}\right) ; \quad\left\{\begin{array}{l}
x^{\prime}=\alpha_{\theta} x+\delta_{\theta} t \\
t^{\prime}=\beta_{\theta} x+\gamma_{\theta} t .
\end{array}\right.
$$

In the following, we consider three particular groups, defined by the conditions

$$
\alpha_{\theta}=\gamma_{\theta}=C_{\theta}, \quad \beta_{\theta}=\frac{\varepsilon}{c} S_{\theta}, \quad \delta_{\theta}=c S_{\theta}
$$

id est by the following matrices

$$
G_{\theta}=\left(\begin{array}{cc}
C_{\theta} & c S_{\theta} \\
\frac{\varepsilon}{c} S_{\theta} & C_{\theta}
\end{array}\right) .
$$

The Galileo group corresponds to

$$
\varepsilon=0, \quad C_{\theta} \equiv 1, \quad S_{\theta} \equiv \theta,
$$

the Lorentz group to

$$
\varepsilon=1, \quad C_{\theta} \equiv \cosh \theta, \quad S_{\theta} \equiv \sinh \theta,
$$

and a third one here called the "circular group" to

$$
\varepsilon=-1, \quad C_{\theta} \equiv \cos \theta, \quad S_{\theta} \equiv \sin \theta .
$$

We remark that we have the elementary relations,

$$
C_{\theta}^{2}-\varepsilon S_{\theta}^{2} \equiv 1, \quad \frac{\mathrm{d} S_{\theta}}{\mathrm{d} \theta} \equiv C_{\theta}, \quad \frac{\mathrm{d} C_{\theta}}{\mathrm{d} \theta} \equiv \varepsilon S_{\theta}, \quad \varepsilon \in\{-1,0,1\} .
$$

- We say here that the system (1) associated with the conservation of entropy

$$
\frac{\partial \eta}{\partial t}+\frac{\partial}{\partial x}(\eta u)=0
$$


is covariant under the action of the group $\mathcal{G}$ if for each $\theta \in \mathbb{R}$ and for each state $W \in \Omega$, we can define a new state $W^{\prime}$ such that after the change of variables (11), the system of equations (1) (17) take the form

$$
\left\{\begin{array}{c}
\frac{\partial W^{\prime}}{\partial t^{\prime}}+\frac{\partial}{\partial x^{\prime}} f\left(W^{\prime}\right)=0 \\
\frac{\partial}{\partial t^{\prime}} \eta\left(W^{\prime}\right)+\frac{\partial}{\partial x^{\prime}}\left(\eta\left(W^{\prime}\right) u\left(W^{\prime}\right)\right)=0
\end{array}\right.
$$

with the same flux function $f(\bullet)$, the same mathematical entropy $\eta(\bullet)$ and the same entropy velocity $u(\bullet)$.

- We present now an elementary calculus. We have the chain rule

$$
\left\{\begin{array}{l}
\frac{\partial}{\partial x}=\frac{\partial x^{\prime}}{\partial x} \frac{\partial}{\partial x^{\prime}}+\frac{\partial t^{\prime}}{\partial x} \frac{\partial}{\partial t^{\prime}}=\alpha_{\theta} \frac{\partial}{\partial x^{\prime}}+\beta_{\theta} \frac{\partial}{\partial t^{\prime}} \\
\frac{\partial}{\partial t}=\frac{\partial x^{\prime}}{\partial t} \frac{\partial}{\partial x^{\prime}}+\frac{\partial t^{\prime}}{\partial t} \frac{\partial}{\partial t^{\prime}}=\delta_{\theta} \frac{\partial}{\partial x^{\prime}}+\gamma_{\theta} \frac{\partial}{\partial t^{\prime}} .
\end{array}\right.
$$

We inject these operators inside the relation (1):

$$
\left(\delta_{\theta} \frac{\partial}{\partial x^{\prime}}+\gamma_{\theta} \frac{\partial}{\partial t^{\prime}}\right) W+\left(\alpha_{\theta} \frac{\partial}{\partial x^{\prime}}+\beta_{\theta} \frac{\partial}{\partial t^{\prime}}\right) f(W)=0
$$

and we put in evidence the new partial derivatives:

$$
\frac{\partial}{\partial t^{\prime}}\left(\gamma_{\theta} W+\beta_{\theta} f(W)\right)+\frac{\partial}{\partial x^{\prime}}\left(\delta_{\theta} W+\alpha_{\theta} f(W)\right)=0 .
$$

We introduce a bijective linear operator $Y_{\theta}$ depending on $\theta \in \mathbb{R}$, acting from $\mathbb{R}^{N}$ into $\mathbb{R}^{N}$ and independent on $x^{\prime}$ and $t^{\prime}$ :

$$
Y_{\theta} \in \mathcal{G} \mathcal{L}\left(\mathbb{R}^{N}\right), \quad \theta \in \mathbb{R} .
$$

After applying $Y_{\theta}$ to the previous equation, we obtain a new conservation law equivalent to (1):

$$
\frac{\partial}{\partial t^{\prime}}\left(\gamma_{\theta} Y_{\theta} \bullet W+\beta_{\theta} Y_{\theta} \bullet f(W)\right)+\frac{\partial}{\partial x^{\prime}}\left(\delta_{\theta} Y_{\theta} \bullet W+\alpha_{\theta} Y_{\theta} \bullet f(W)\right)=0 .
$$

A sufficient condition to establish the first equation of $(18)$ is to identify the arguments of the two partial derivatives $\partial_{t^{\prime}}$ and $\partial_{x^{\prime}}$ in the previous equation:

$$
\left\{\begin{array}{l}
W^{\prime}=\gamma_{\theta} Y_{\theta} \bullet W+\beta_{\theta} Y_{\theta} \bullet f(W) \\
f\left(W^{\prime}\right)=\delta_{\theta} Y_{\theta} \bullet W+\alpha_{\theta} Y_{\theta} \bullet f(W) .
\end{array}\right.
$$

The relation 21 can be written in a more compact form:

$$
\left(\begin{array}{c}
f\left(W^{\prime}\right) \\
W^{\prime}
\end{array}\right)=G_{\theta} Y_{\theta} \bullet\left(\begin{array}{c}
f(W) \\
W
\end{array}\right) \equiv G_{\theta}\left(\begin{array}{c}
Y_{\theta} \bullet f(W) \\
Y_{\theta} \bullet W
\end{array}\right) .
$$

Under the "linearity hypothesis" $(19)$, the relation (22) gives geometrical constraints for the transformation $\Omega \ni W \longmapsto W^{\prime} \in \Omega$ associated with the action of the group $\mathcal{G}$.

- We make an analogous calculus for the second equation of (18) relative to entropy. After the transformation of partial derivatives, the relation (17) takes the form

$$
\left(\delta_{\theta} \frac{\partial}{\partial x^{\prime}}+\gamma_{\theta} \frac{\partial}{\partial t^{\prime}}\right) \eta(W)+\left(\alpha_{\theta} \frac{\partial}{\partial x^{\prime}}+\beta_{\theta} \frac{\partial}{\partial t^{\prime}}\right)(\eta(W) u(W))=0
$$




\section{FranÇOIS DUBOIS}

and can be written as

$$
\frac{\partial}{\partial t^{\prime}}\left(\gamma_{\theta} \eta(W)+\beta_{\theta} \eta(W) u(W)\right)+\frac{\partial}{\partial x^{\prime}}\left(\delta_{\theta} \eta(W)+\alpha_{\theta} \eta(W) u(W)\right)=0 .
$$

This is a scalar conservation law. This equation is identical to the second equation of (18) if the following sufficient conditions are satisfied:

$$
\left\{\begin{array}{cl}
\eta\left(W^{\prime}\right) & =\gamma_{\theta} \eta(W)+\beta_{\theta} \eta(W) u(W) \\
\eta\left(W^{\prime}\right) u\left(W^{\prime}\right) & =\delta_{\theta} \eta(W)+\alpha_{\theta} \eta(W) u(W) .
\end{array}\right.
$$

With a notation that makes the action of the group $\mathcal{G}$ explicit:

$$
\left(\begin{array}{c}
\eta\left(W^{\prime}\right) u\left(W^{\prime}\right) \\
\eta\left(W^{\prime}\right)
\end{array}\right)=G_{\theta}\left(\begin{array}{c}
\eta(W) u(W) \\
\eta(W)
\end{array}\right) .
$$

\section{- Proposition 2. Linear representation}

If the relation $(22)$ is satisfied for every $\theta \in \mathbb{R}$, then the application $\mathcal{G} \ni G_{\theta} \longmapsto Y_{\theta} \in$ $\mathcal{G} \mathcal{L}\left(\mathbb{R}^{N}\right)$ is a linear representation of the group $\mathcal{G}$ :

$$
Y_{\theta+\theta^{\prime}}=Y_{\theta} \bullet Y_{\theta^{\prime}}, \quad \theta, \theta^{\prime} \in \mathbb{R} .
$$

\section{Proof of Proposition 2.}

We first consider the relation $(22)$ and an analogous relation obtained by replacing the variable $\theta$ by the variable $\theta^{\prime}$ :

$$
\left(\begin{array}{c}
f\left(W^{\prime \prime}\right) \\
W^{\prime \prime}
\end{array}\right)=G_{\theta}^{\prime} Y_{\theta}^{\prime} \bullet\left(\begin{array}{c}
f\left(W^{\prime}\right) \\
W^{\prime}
\end{array}\right) \equiv G_{\theta}^{\prime}\left(\begin{array}{c}
Y_{\theta}^{\prime} \bullet f\left(W^{\prime}\right) \\
Y_{\theta}^{\prime} \bullet W^{\prime}
\end{array}\right)
$$

We compose this relation with 22 and we obtain

$$
\left(\begin{array}{c}
f\left(W^{\prime \prime}\right) \\
W^{\prime \prime}
\end{array}\right)=G_{\theta^{\prime}} G_{\theta}\left(\begin{array}{c}
Y_{\theta^{\prime}} \bullet Y_{\theta} \bullet f(W) \\
Y_{\theta^{\prime}} \bullet Y_{\theta} \bullet W
\end{array}\right) .
$$

When we apply the relation 22 with the argument $\theta+\theta^{\prime}$, we have directly

$$
\left(\begin{array}{c}
f\left(W^{\prime \prime}\right) \\
W^{\prime \prime}
\end{array}\right)=G_{\theta+\theta^{\prime}}\left(\begin{array}{c}
Y_{\theta+\theta^{\prime}} \bullet f(W) \\
Y_{\theta+\theta^{\prime}} \bullet W
\end{array}\right) .
$$

We look precisely to the two previous relations. The scalars $\alpha_{\theta}, \beta_{\theta}, \gamma_{\theta}$ and $\delta_{\theta}$ commute with the linear operator $Y_{\theta}$ and we obtain the relation (26) because the two previous equalities are satisfied for an arbitrary state $W$.

\section{4) Manifold of null velocity}

- In the following we denominate by $\Omega_{0}$ the set of states with a velocity equal to zero. Because the mapping $\Omega \ni W \longmapsto u(W) \in \mathbb{R}$ is scalar, it is natural to suppose that $\Omega_{0}$ is a manifold in $\mathbb{R}^{N}$ of codimension one. We observe also that the flux function $f(\bullet)$ is reduced to its thermodynamic contribution $g(\bullet)$ on the manifold of null velocity. Our program is to construct the states $W$, the flux function $f(\bullet)$ and the mathematical entropy $\eta(\bullet)$ when we suppose that the flux function $\Omega_{0} \ni W_{0} \longmapsto g_{0}\left(W_{0}\right) \in \mathbb{R}^{N}$ and the mathematical entropy $\Omega_{0} \ni W_{0} \longmapsto \sigma\left(W_{0}\right) \in \mathbb{R}$ are given on the manifold of null velocity. 
The idea is to use the group invariance to link a given state $W$ with an appropriate state $W_{0}$ on the manifold $\Omega_{0}$ as presented in Figure 1 . The conditions (22) and (25) are geometric constraints that will allow essentially to solve the problem.

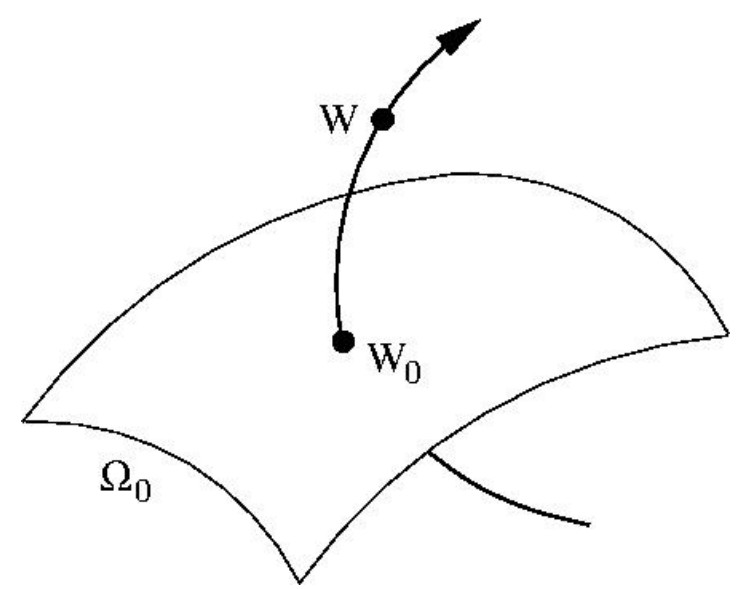

Figure 1. Fiber above the manifold of null velocity $\Omega_{0}$. Thanks to the action of the group, a state $W_{0}$ of null velocity $\Omega_{0}$ generates a current state $W$.

\section{- Proposition 3. Velocity field}

With the hypothesis (21) and if the mathematical entropy $\sigma$ on the manifold of null velocity is not the null function, the velocity field $u(W)$ is necessarily given by the relation

$$
u(W)=\frac{\delta_{\theta}}{\gamma_{\theta}} .
$$

\section{Proof of Proposition 3.}

We consider a given state $W_{0}$ on the manifold $\Omega_{0}$ and a running state $W \in \Omega$ obtained by the relations (21). With the actual notations, we have

$$
W=\gamma_{\theta} Y_{\theta} \bullet W_{0}+\beta_{\theta} Y_{\theta} \bullet g_{0}\left(W_{0}\right), \quad f(W)=\delta_{\theta} Y_{\theta} \bullet W_{0}+\alpha_{\theta} Y_{\theta} \bullet g_{0}\left(W_{0}\right) .
$$

The analogous relations (24) relative to the entropy take a simple form

$$
\eta(W)=\gamma_{\theta} \sigma\left(W_{0}\right), \quad \eta(W) u(W)=\delta_{\theta} \sigma\left(W_{0}\right) .
$$

because the velocity is also null on $\Omega_{0}$. We consider a state $W_{0}$ such that $\sigma\left(W_{0}\right) \neq 0$. If $\gamma_{\theta}$ is null, then $\eta(W)=0$ is null and $\delta_{\theta}=0$ because $\sigma\left(W_{0}\right) \neq 0$. This contradicts the fact that the matrix defining $G_{\theta}$ by the relation $(10)$ is invertible. Then $\gamma_{\theta} \neq 0$ and the relation (27) is obtained by taking the ratio of the two relations of (29).

- With the example proposed in (12, we have

$$
u=c \frac{S_{\theta}}{C_{\theta}} .
$$

We observe that we have also established the following property: 


\section{FranÇOIS DUBOIS}

\section{- Proposition 4. Entropy field}

With the above hypotheses, if the state $W$ is given from $W_{0} \in \Omega_{0}$ with the help of the relations (28), we have necessarily

$$
\eta(W)=\gamma_{\theta} \sigma\left(W_{0}\right) .
$$

- Hypothesis 1. The null-velocity manifold $\Omega_{0}$ is included in an hyperplane We remark that if a convex restriction of the mathematical entropy $\Omega_{0} \ni W_{0} \longmapsto \sigma\left(W_{0}\right) \in$ $\mathbb{R}$ is given on the manifold $\Omega_{0}$, a natural hypothesis is to suppose $\Omega_{0}$ convex. We will do this hypothesis in the following. Moreover, we suppose that $\Omega_{0}$ is flat; this is expressed by our hypothesis 1 .

\section{- Proposition 5. Thermodynamic flux}

With the above framework (28), the thermodynamic flux $g(W)$ is given from the datum $g_{0}\left(W_{0}\right)$ according to

$$
g(W)=\left(\alpha_{\theta}-u(W) \beta_{\theta}\right) Y_{\theta} \bullet g_{0}\left(W_{0}\right) .
$$

\section{Proof of Proposition 5.}

The second relation of (28) and the definition of the thermodynamic flux (8) allows us to evaluate the function $g(\bullet)$. We have

$$
\begin{aligned}
g(W) & =f(W)-u(W) W \\
& =\delta_{\theta} Y_{\theta} \bullet W_{0}+\alpha_{\theta} Y_{\theta} \bullet g_{0}\left(W_{0}\right)-u(W)\left(\gamma_{\theta} Y_{\theta} \bullet W_{0}+\beta_{\theta} Y_{\theta} \bullet g_{0}\left(W_{0}\right)\right) \\
& =\left(\alpha_{\theta}-u(W) \beta_{\theta}\right) Y_{\theta} \bullet g_{0}\left(W_{0}\right) \quad \text { due to } 27
\end{aligned}
$$

and the relation 32 is established.

- For the groups proposed in $(12)$, we have $\alpha_{\theta}-u(W) \beta_{\theta}=C_{\theta}-c \frac{S_{\theta}}{C_{\theta}} \frac{\varepsilon}{c} S_{\theta}=\frac{1}{C_{\theta}}$ due to (16). Then the relation (32) takes the form

$$
g(W)=\frac{1}{C_{\theta}} Y_{\theta} \bullet g_{0}\left(W_{0}\right) .
$$

\section{5) Entropy variables}

- Even if the state and the entropy are only partially known with the help of relations (28) and (31), its jacobian $\varphi$ introduced in (4) relative to the conserved variables $W$ can be essentially determined. We first consider the dynamical equations present in this study. First, the relation

$$
G_{\theta+\theta^{\prime}}=G_{\theta} \bullet G_{\theta^{\prime}}
$$

and the relation $(26)$ for the matrices $Y_{\theta}$ can be differentiated relatively to $\theta$. We have

$$
\frac{\mathrm{d} G_{\theta}}{\mathrm{d} \theta}=G_{0}^{\prime} G_{\theta}=G_{\theta} G_{0}^{\prime}, \quad \frac{\mathrm{d} Y_{\theta}}{\mathrm{d} \theta}=Y_{0}^{\prime} Y_{\theta}=Y_{\theta} Y_{0}^{\prime} .
$$

With the choice (12) of one classical group, we have

$$
G_{0}^{\prime}=\left(\begin{array}{cc}
0 & c \\
\varepsilon & 0 \\
\bar{c} & 0
\end{array}\right)
$$


and we can precise how to extend the differential relatations (34) and (35).

\section{- Proposition 6. Differential relations for the entropy variables}

With the above context, we have

$$
G_{0}^{\prime}\left(\begin{array}{c}
u \eta^{*}+\varphi g \\
\eta^{*}
\end{array}\right) \mathrm{d} \theta+\left(\begin{array}{c}
\varphi Y_{0}^{\prime} f(W) \\
\varphi Y_{0}^{\prime} W
\end{array}\right) \mathrm{d} \theta+G_{\theta}\left(\begin{array}{c}
\varphi Y_{\theta} \mathrm{d} g_{0} \\
\varphi Y_{\theta} \mathrm{d} W_{0}-\mathrm{d} \sigma
\end{array}\right)=0
$$

\section{Proof of Proposition 6.}

We write the relation (28) under a matricial form

$$
\left(\begin{array}{c}
f(W) \\
W
\end{array}\right)=G_{\theta}\left(\begin{array}{c}
Y_{\theta} g_{0}\left(W_{0}\right) \\
Y_{\theta} W_{0}
\end{array}\right)
$$

We differentiate the relation (38), taking into account the two contributions relative to the group $\mathcal{G}$ on one hand and along the manifold $\Omega_{0}$ on the other hand, as illustrated on the figure 1 . Then

$$
\left(\begin{array}{c}
\mathrm{d} f \\
\mathrm{~d} W
\end{array}\right)=G_{0}^{\prime} G_{\theta}\left(\begin{array}{cc}
Y_{\theta} & g_{0} \\
Y_{\theta} & W_{0}
\end{array}\right) \mathrm{d} \theta+G_{\theta}\left(\begin{array}{ccc}
Y_{0}^{\prime} & Y_{\theta} & g_{0} \\
Y_{0}^{\prime} & Y_{\theta} & W_{0}
\end{array}\right) \mathrm{d} \theta+G_{\theta}\left(\begin{array}{cc}
Y_{\theta} & \mathrm{d} g_{0} \\
Y_{\theta} & \mathrm{d} W_{0}
\end{array}\right)
$$

and because

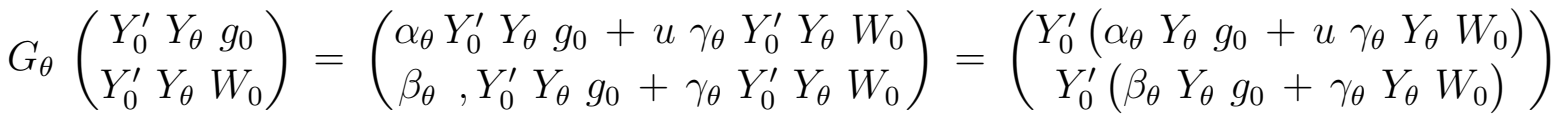

$$
\begin{aligned}
& =\left(\begin{array}{c}
Y_{0}^{\prime} f(W) \\
Y_{0}^{\prime} W
\end{array}\right)
\end{aligned}
$$

we have

$$
\left(\begin{array}{c}
\mathrm{d} f \\
\mathrm{~d} W
\end{array}\right)=G_{0}^{\prime}\left(\begin{array}{c}
f \\
W
\end{array}\right) \mathrm{d} \theta+\left(\begin{array}{cc}
Y_{0}^{\prime} & f \\
Y_{0}^{\prime} & W
\end{array}\right) \mathrm{d} \theta+G_{\theta}\left(\begin{array}{cc}
Y_{\theta} \mathrm{d} g_{0} \\
Y_{\theta} \mathrm{d} W_{0}
\end{array}\right) .
$$

We multiply each line of the relation $(39)$ by the line vector $\varphi$. We remark that

$$
\begin{aligned}
\varphi G_{0}^{\prime}\left(\begin{array}{c}
\mathrm{d} f \\
\mathrm{~d} W
\end{array}\right) & =\varphi\left(\begin{array}{cc}
\alpha_{0}^{\prime} & (u \gamma)_{0}^{\prime} \\
\beta_{0}^{\prime} & \gamma_{0}^{\prime}
\end{array}\right)\left(\begin{array}{c}
\mathrm{d} f \\
\mathrm{~d} W
\end{array}\right)=\varphi\left(\begin{array}{c}
\alpha_{0}^{\prime} f+(u \gamma)_{0}^{\prime} W \\
\beta_{0}^{\prime} f+\gamma_{0}^{\prime} W
\end{array}\right) \\
& =\left(\begin{array}{c}
\alpha_{0}^{\prime} \varphi f+(u \gamma)_{0}^{\prime} \varphi W \\
\beta_{0}^{\prime} \varphi f+\gamma_{0}^{\prime} \varphi W
\end{array}\right)=G_{0}^{\prime}\left(\begin{array}{c}
\varphi f \\
\varphi W
\end{array}\right)
\end{aligned}
$$

and in a similar way,

$$
\begin{aligned}
\varphi G_{\theta} Y_{\theta}\left(\begin{array}{c}
\mathrm{d} g_{0} \\
\mathrm{~d} W_{0}
\end{array}\right) & =\varphi\left(\begin{array}{c}
\alpha_{\theta} Y_{\theta} \mathrm{d} g_{0}+(u \gamma)_{\theta} Y_{\theta} \mathrm{d} W_{0} \\
\beta_{\theta} Y_{\theta} \mathrm{d} g_{0}+\gamma_{\theta} Y_{\theta} \mathrm{d} W_{0}
\end{array}\right) \\
& =\left(\begin{array}{c}
\alpha_{\theta} \varphi Y_{\theta} \mathrm{d} g_{0}+(u \gamma)_{\theta} \varphi Y_{\theta} \mathrm{d} W_{0} \\
\beta_{\theta} \varphi Y_{\theta} \mathrm{d} g_{0}+\gamma_{\theta} \varphi Y_{\theta} \mathrm{d} W_{0}
\end{array}\right)=G_{\theta}\left(\begin{array}{c}
\varphi Y_{\theta} \mathrm{d} g_{0} \\
\varphi Y_{\theta} \mathrm{d} W_{0}
\end{array}\right) .
\end{aligned}
$$

Then

$$
\left(\begin{array}{c}
\varphi \mathrm{d} f \\
\varphi \mathrm{d} W
\end{array}\right)=G_{0}^{\prime}\left(\begin{array}{cc}
\varphi & f \\
\varphi & W
\end{array}\right) \mathrm{d} \theta+\left(\begin{array}{ccc}
\varphi & Y_{0}^{\prime} & f \\
\varphi & Y_{0}^{\prime} & W
\end{array}\right) \mathrm{d} \theta+G_{\theta}\left(\begin{array}{ccc}
\varphi & Y_{\theta} \mathrm{d} g_{0} \\
\varphi & Y_{\theta} & \mathrm{d} W_{0}
\end{array}\right)
$$

- We develop the same calculus for the entropy. We start with a matricial form of the relation 29 :

$$
\left(\begin{array}{c}
\eta u \\
\eta
\end{array}\right)=G_{\theta}\left(\begin{array}{l}
0 \\
\sigma
\end{array}\right)
$$




\section{FrançOIS Dubois}

Then

$$
\left(\begin{array}{c}
\mathrm{d}(\eta u) \\
\mathrm{d} \eta
\end{array}\right)=G_{0}^{\prime}\left(\begin{array}{c}
\eta u \\
\eta
\end{array}\right) \mathrm{d} \theta+G_{\theta}\left(\begin{array}{c}
0 \\
\mathrm{~d} \sigma
\end{array}\right)
$$

We make the difference between the relations $(42)$ and (40). Due to (3) and (4) the left hand side is equal to zero! Then the right hand side can be written as

$$
G_{0}^{\prime}\left(\begin{array}{c}
\varphi f-\eta u \\
\varphi W-\eta
\end{array}\right) \mathrm{d} \theta+\left(\begin{array}{ccc}
\varphi & Y_{0}^{\prime} & f \\
\varphi & Y_{0}^{\prime} & W
\end{array}\right) \mathrm{d} \theta+G_{\theta}\left(\begin{array}{c}
\varphi Y_{\theta} \mathrm{d} g_{0} \\
\varphi Y_{\theta} \mathrm{d} W_{0}-\mathrm{d} \sigma
\end{array}\right) .
$$

The relation (37) is now easily obtained by the explicitation of the dual entropy introduced in (5) and the thermodynamic flux defined in (8).

\section{- Proposition 7. Constraints for the entropy variables}

With the above framework, if we suppose moreover that the derivative of the group $\mathcal{G}$ for $\theta=0$ is given by the relation (36), we have the following relations for the entropy variables

$$
\begin{gathered}
c \eta^{*}+\varphi Y_{0}^{\prime} f(W)=0 \\
\frac{\varepsilon}{c}\left(u \eta^{*}+\varphi g(W)\right)+\varphi Y_{0}^{\prime} W=0 \\
\varphi Y_{\theta} \mathrm{d} g_{0}=0 \\
\varphi Y_{\theta} \mathrm{d} W_{0}-\mathrm{d} \sigma=0 .
\end{gathered}
$$

\section{Proof of Proposition 7.}

With the matrix $G_{0}^{\prime}$ chosen according to $(36)$, the relation $(37)$ becomes

$$
\left(\begin{array}{c}
c \eta^{*} \\
\frac{\varepsilon}{c}\left(u \eta^{*}+\varphi g(W)\right)
\end{array}\right) \mathrm{d} \theta+\left(\begin{array}{c}
\varphi Y_{0}^{\prime} f(W) \\
\varphi Y_{0}^{\prime} W
\end{array}\right) \mathrm{d} \theta+G_{\theta}\left(\begin{array}{c}
\varphi Y_{\theta} \mathrm{d} g_{0} \\
\varphi Y_{\theta} \mathrm{d} W_{0}-\mathrm{d} \sigma
\end{array}\right)=0
$$

and the relations (43) to (46) are clear.

- The relation (46) can be expressed with the help of the gradient $\varphi_{0}$ of the entropy restricted on the manifold $\Omega_{0}$ :

$$
\mathrm{d} \sigma \equiv \varphi_{0} \bullet \mathrm{d} W_{0} .
$$

Then the relation 46 express that the difference $\varphi Y_{\theta}-\varphi_{0}$ belongs to the polar set $\Omega_{0}^{0}$ of $\Omega_{0}$ : we can write $\varphi Y_{\theta}-\varphi_{0}=\mu r_{0}$ for some scalar $\mu$ and some non-null linear form $r_{0}$ identically equal to zero on $\Omega_{0}$. We make also a new hypothesis.

- Hypothesis 2. The entropy $\sigma$ and the thermodynamic flux $g_{0}$ are weakly decoupled on the hyperplane $\Omega_{0}$

In other terms,

$$
\left(\varphi_{0}, \mathrm{~d} g_{0} \bullet \rho_{0}\right)=0, \quad \forall \rho_{0} \in \Omega_{0} .
$$

- Proposition 8. Explicitation of the entropy variables

We make the hypotheses 1 and 2 and suppose moreover

$$
\exists \rho_{0} \in \Omega_{0}, \quad \mathrm{~d} g_{0} \bullet \rho_{0} \neq 0 .
$$


Then the relations $(43)$ to 446 imply a simple form for the entropy variables:

$$
\varphi Y_{\theta}=\varphi_{0} .
$$

\section{Proof of Proposition 8.}

Consider an arbitrary vector $\rho_{0} \in \Omega_{0}$. When we apply the relation $\varphi Y_{\theta}-\varphi_{0}=\mu r_{0}$ established previously to the vector $\mathrm{d} g_{0} \bullet \rho_{0}$, we find zero due to the constraint 45 and the hypothesis 2 with (48). Then $\mu<r_{0}, \mathrm{~d} g_{0} \bullet \rho_{0}>=0$. With the choice of $\rho_{0}$ suggested in (49), the duality product $\left\langle r_{0}, \mathrm{~d} g_{0} \bullet \rho_{0}\right\rangle$ is not null. So $\mu=0$ and the relation (50) is established.

- Hypothesis 3. The entropy $\sigma$ and the thermodynamic flux $g_{0}$ are strongly decoupled on the hyperplane $\Omega_{0}$

In other terms,

$$
\varphi_{0}\left(W_{0}\right) \bullet g_{0}\left(\widetilde{W_{0}}\right)=0, \quad \forall W_{0}, \widetilde{W_{0}} \in \Omega_{0} .
$$

We observe that this strong hypothesis (51) implies (48) by differentiation relatively to the variable $\widetilde{W}_{0}$ in $\Omega_{0}$. In consequence, we have a new property for the entropy variables.

- Proposition 9. The entropy variables are in the polar set of the thermo flux

We make the hypotheses 1 and 3 and suppose also the technical condition 49 proposed previously. Then we have a complete decoupling:

$$
\varphi(W) \bullet g(\widetilde{W}) \equiv 0 \quad \forall W, \widetilde{W} \in \Omega
$$

\section{Proof of Proposition 9.}

We just have to precise the expression (33) of the thermodynamic flux. Then we have

$$
\varphi(W) \bullet g(\widetilde{W})=\varphi(W) Y_{\theta} Y_{-\theta} g(\widetilde{W})=\varphi_{0}\left(W_{0}\right) \frac{1}{C_{\theta}} g_{0}\left(\widetilde{W_{0}}\right)=0
$$

due to 52 .

\section{- Proposition 10. Constraints for the representation $Y$}

Under the same hypotheses that the ones done for Proposition 9, we have

$$
\begin{aligned}
& c \eta^{*}+C_{\theta} \varphi_{0} Y_{0}^{\prime} g_{0}=0 \\
& \varphi_{0} Y_{0}^{\prime} W_{0}=0 \quad \text { on } \Omega_{0} .
\end{aligned}
$$

\section{Proof of Proposition 10.}

We first have the following calculus:

$$
\varphi Y_{0}^{\prime} g=\varphi Y_{\theta} Y_{-\theta} Y_{0}^{\prime} g=\varphi Y_{\theta} Y_{0}^{\prime} Y_{-\theta} g=\varphi_{0} Y_{0}^{\prime} \frac{1}{C_{\theta}} g_{0}=\frac{1}{C_{\theta}} \varphi_{0} Y_{0}^{\prime} g_{0} .
$$

Then we multiply the equation (44) by the opposite of the velocity $u$ and we add it to (43). With the definition (8) of the thermodynamic flux, we have, taking into account the "trigonometrical relations" (16) and the expression (27) of the velocity:

$0=\eta^{*}\left(c-\frac{\varepsilon u^{2}}{c}\right)+\varphi Y_{0}^{\prime} g=\eta^{*}\left(c-\frac{\varepsilon c S_{\theta}^{2}}{C_{\theta}^{2}}\right)+\frac{1}{C_{\theta}} \varphi_{0} Y_{0}^{\prime} g_{0}=\frac{c \eta^{*}}{C_{\theta}^{2}}+C_{\theta} \varphi_{0} Y_{0}^{\prime} g_{0}$ 


\section{FranÇOIS DUBOIS}

and the relation $(53)$ is established. We report now the expression $(28)$ of a current state $W$ inside the equation (44):

$$
\begin{aligned}
0 & =\frac{\varepsilon}{c} u \eta^{*}+\varphi Y_{0}^{\prime} Y_{\theta} \bullet\left(C_{\theta} W_{0}+\frac{\varepsilon}{c} S_{\theta} g_{0}\right)=C_{\theta} \varphi Y_{\theta} Y_{0}^{\prime} W_{0}+\frac{\varepsilon}{c}\left(u \eta^{*}+S_{\theta} \varphi Y_{\theta} Y_{0}^{\prime} g_{0}\right) \\
& =C_{\theta} \varphi Y_{\theta} Y_{0}^{\prime} W_{0}+\frac{\varepsilon}{c}\left(u \eta^{*}-S_{\theta} \frac{c}{C_{\theta}} \eta^{*}\right)=C_{\theta} \varphi Y_{\theta} Y_{0}^{\prime} W_{0}+\varepsilon \eta^{*}\left(\frac{u}{c}-\frac{S_{\theta}}{C_{\theta}}\right) \\
& =C_{\theta} \varphi Y_{\theta} Y_{0}^{\prime} W_{0}
\end{aligned}
$$

and the relation (54) follows from (50).

\section{- Proposition 11. Dual entropy}

Under the hypotheses proposed at Proposition 9, we denote by $\sigma^{*}$ the dual of the entropy $\sigma$ restricted on the manifold of null velocity:

$$
\sigma^{*} \equiv \varphi_{0} \bullet W_{0}-\sigma\left(W_{0}\right) \quad \text { on } \Omega_{0}, \quad \text { with } \quad \varphi_{0}=\sigma^{\prime}\left(W_{0}\right) \text {. }
$$

Then we have

$$
\eta^{*}=C_{\theta} \sigma^{*}
$$

and the relation $(53)$ takes the form

$$
c \sigma^{*}+\varphi_{0} Y_{0}^{\prime} g_{0}=0 \quad \text { on } \Omega_{0} .
$$

\section{Proof of Proposition 11.}

We have, thanks to (5), (31), (38) and (50) :

$$
\eta^{*}=\varphi \bullet W-\eta(W)=\varphi_{0} \bullet Y_{-\theta} Y_{\theta}\left(C_{\theta} W_{0}+\frac{\varepsilon}{c} S_{\theta} g_{0}\right)-C_{\theta} \sigma=C_{\theta}\left(\varphi_{0} \bullet W_{0}-\sigma\right)
$$

due to Hypothesis 3 (relation (51)). The end of the proof is clear.

\section{6) Hyperbolic systems of order two}

- If we suppose $N=2$, the matrix $Y_{\theta}$ is a two by two matrix. We suppose here that this matrix has the same algebaic form that the form $(12)$ suggested for $G_{\theta}$. We set for this contribution

$$
Y_{\theta}=\left(\begin{array}{cc}
\widetilde{C}_{\theta} & \widetilde{\varepsilon} \\
\widetilde{S}_{a} & \widetilde{S}_{\theta} \\
\widetilde{C}_{\theta} & \widetilde{C}_{\theta}
\end{array}\right), \quad \theta \in \mathbb{R}, \quad a>0 .
$$

In the relation $(58)$, the functions $\widetilde{S}_{\theta}$ and $\widetilde{C}_{\theta}$ are analogous to the ones proposed in 13 , (14), (15) and (16) for $S_{\theta}$ and $C_{\theta}$. We have in particular

$$
\widetilde{C}_{\theta}^{2}-\widetilde{\varepsilon} \widetilde{S}_{\theta}^{2} \equiv 1 \text {. }
$$

The nilpotent representation of the group $\mathcal{G}$ corresponds to $\widetilde{\varepsilon}=0$, the hyperbolic representation to $\widetilde{\varepsilon}=1$ and the elliptic one to $\widetilde{\varepsilon}=-1$. The derivative $Y_{\theta}^{\prime}$ of the matrix $Y_{\theta}$ proposed in (58) at $\theta=0$ is given by

$$
Y_{0}^{\prime}=\left(\begin{array}{cc}
0 & \frac{\widetilde{\varepsilon}}{a} \\
a & 0
\end{array}\right)
$$


- We particularize the system by a simple choice for the null-velocity manifold. As in our previous work [6], we suppose

$$
W_{0}=\left(\begin{array}{c}
\rho_{0} \\
0
\end{array}\right) \in \Omega_{0}
$$

and

$$
g_{0}\left(W_{0}\right)=\left(\begin{array}{c}
0 \\
p_{0}
\end{array}\right) \quad \text { for } W_{0} \in \Omega_{0} .
$$

With this choice, the hypotheses 2 and 3 are satisfied. The variable $p_{0}$ in the right hand side of the relation 62 is the thermodynamic pressure.

- Proposition 12. Pressure as a thermodynamic variable

With the above hypotheses, we have the relation

$$
\sigma^{*}+\frac{\widetilde{\varepsilon}}{a c} \sigma^{\prime} p_{0}=0
$$

\section{Proof of Proposition 12.}

Just write the relation (57) with the help of 60 and 61 :

$c \sigma^{*}+\varphi_{0} Y_{0}^{\prime} g_{0}=c \sigma^{*}+\left(\begin{array}{cc}\sigma^{\prime} & 0\end{array}\right)\left(\begin{array}{cc}0 & \frac{\widetilde{\varepsilon}}{a} \\ a & 0\end{array}\right)\left(\begin{array}{c}0 \\ p_{0}\end{array}\right)=c \sigma^{*}+\frac{\widetilde{\varepsilon}}{a} \sigma^{\prime} p_{0}$

and the relation 63 is established.

- The nilpotent case $(\widetilde{\varepsilon}=0)$ has no interest when $N=2$ because the dual of the entropy is necessary null, as we have remarked in [6] in the particular case of the Galileo group $(\varepsilon=0)$. We can explicit the first relations of $(28)$. With the notation

$$
W \equiv\left(\begin{array}{l}
\rho \\
J
\end{array}\right)
$$

we have

$$
\rho=C_{\theta} \widetilde{C}_{\theta} \rho_{0}+\frac{\varepsilon \widetilde{\varepsilon}}{a c} S_{\theta} \widetilde{S}_{\theta} p_{0}, \quad \frac{J}{a}=C_{\theta} \widetilde{S}_{\theta} \rho_{0}+\frac{\varepsilon}{a c} S_{\theta} \widetilde{C}_{\theta} p_{0}
$$

- In the case of Galileo group, we have $\varepsilon=0, C_{\theta}=1, S_{\theta}=\theta$ and $u=c \theta$. The explicitation of the parameter $\theta$ and the state of null velocity $\rho_{0}$ is simple by resolution of the system 65). We have

$$
\rho=\widetilde{C}_{\theta} \rho_{0}, \quad \frac{J}{a}=\widetilde{S}_{\theta} \rho_{0}, \quad \varepsilon=0, \quad \text { Galileo group. }
$$

In the hyperbolic case, we have

$$
\tanh \theta=\frac{J}{a \rho}, \quad u=c \theta, \quad \varepsilon=0, \widetilde{\varepsilon}=1
$$

and in the elliptic one,

$$
\operatorname{tg} \theta=\frac{J}{a \rho}, \quad u=c \theta, \quad \varepsilon=0, \widetilde{\varepsilon}=-1
$$

as we have explained in [6]. 


\section{FranÇOIS DUBOIS}

- In the following, we focus on hyperbolic $(\widetilde{\varepsilon}=1)$ and elliptic $(\widetilde{\varepsilon}=-1)$ representations of the Lorentz $(\varepsilon=1)$ and circular $(\varepsilon=-1)$ groups. With other words,

$$
\varepsilon^{2}=\widetilde{\varepsilon}^{2}=1 \text {. }
$$

We can replace the pressure $p_{0}$ with an appropriate thermodynamic quantity because

$$
\frac{\sigma^{*}}{\sigma^{\prime}}=\frac{\rho_{0} \sigma^{\prime}-\sigma}{\sigma^{\prime}}=\rho_{0}-\frac{\sigma}{\sigma^{\prime}} .
$$

Then the relations 65 take the form

(67) $\rho=\left(C_{\theta} \widetilde{C}_{\theta}-\varepsilon S_{\theta} \widetilde{S}_{\theta}\right) \rho_{0}+\varepsilon S_{\theta} \widetilde{S}_{\theta} \frac{\sigma}{\sigma^{\prime}}, \quad \frac{J}{a}=\left(C_{\theta} \widetilde{S}_{\theta}-\varepsilon \widetilde{\varepsilon} S_{\theta} \widetilde{C}_{\theta}\right) \rho_{0}+\varepsilon \widetilde{\varepsilon} S_{\theta} \widetilde{C}_{\theta} \frac{\sigma}{\sigma^{\prime}}$.

For the Lorentz $(\varepsilon=1)$ or the circular $(\varepsilon=-1)$ group, the resolution of the system (67) with unknowns $\theta$ and $\rho_{0}$ is absolutly non trivial. In order to be able to differentiate the solution $\left(\theta, \rho_{0}\right)$ relatively to $(\rho, J)$, we have the following result.

\section{- Proposition 13. Jacobian}

We denote by $\zeta_{0}$ the partial derivative of the ratio $\sigma / \sigma^{\prime}$ relatively to the dentity $\rho_{0}$ at rest :

$$
\zeta_{0} \equiv \frac{\partial}{\partial \rho_{0}}\left(\frac{\sigma}{\sigma^{\prime}}\right)
$$

If the hypothesis (66) is satisfied and if the following jacobian determinant

$$
\Delta \equiv \frac{\partial(\rho, J)}{\partial\left(\theta, \rho_{0}\right)}=a \frac{\sigma}{\sigma^{\prime}}\left[(\varepsilon \widetilde{\varepsilon}-1) C_{\theta}^{2} \frac{\sigma^{*}}{\sigma}+\widetilde{\varepsilon} S_{\theta}^{2} \zeta_{0}-\left(C_{\theta}^{2}+\widetilde{\varepsilon} S_{\theta}^{2}\right)\right]
$$

is not null, the solution $\left(\theta, \rho_{0}\right)$ of the system (67) is locally unique and we can differentiate the parameters $\theta$ and $\rho_{0}$ relatively to the state $W$ introduced in (64).

\section{Proof of Proposition 13.}

We have

$$
\left\{\begin{aligned}
\frac{\partial \rho}{\partial \theta} & =(\widetilde{\varepsilon}-\varepsilon) C_{\theta} \widetilde{S}_{\theta} \rho_{0}+\varepsilon\left(C_{\theta} \widetilde{S}_{\theta}+S_{\theta} \widetilde{C}_{\theta}\right) \frac{\sigma}{\sigma^{\prime}} \\
\frac{\partial \rho}{\partial \rho_{0}} & =\left(C_{\theta} \widetilde{C}_{\theta}-\varepsilon S_{\theta} \widetilde{S}_{\theta}\right)+\varepsilon S_{\theta} \widetilde{S}_{\theta} \zeta_{0} \\
\frac{1}{a} \frac{\partial J}{\partial \theta} & =(1-\varepsilon \widetilde{\varepsilon}) C_{\theta} \widetilde{C}_{\theta} \rho_{0}+\left(\varepsilon \widetilde{\varepsilon} C_{\theta} \widetilde{C}_{\theta}+\varepsilon S_{\theta} \widetilde{S}_{\theta}\right) \frac{\sigma}{\sigma^{\prime}} \\
\frac{1}{a} \frac{\partial J}{\partial \rho_{0}} & =\left(C_{\theta} \widetilde{S}_{\theta}-\varepsilon \widetilde{\varepsilon} S_{\theta} \widetilde{C}_{\theta}\right)+\varepsilon \widetilde{\varepsilon} S_{\theta} \widetilde{C}_{\theta} \zeta_{0} .
\end{aligned}\right.
$$

Then the determinant $\Delta \equiv \frac{\partial(\rho, J)}{\partial\left(\theta, \rho_{0}\right)}$ can be evaluated as follows :

$$
\begin{aligned}
\Delta & =\frac{\partial \rho}{\partial \theta} \frac{\partial J}{\partial \rho_{0}}-\frac{\partial \rho}{\partial \rho_{0}} \frac{\partial J}{\partial \theta} \\
= & a\left[\left((\widetilde{\varepsilon}-\varepsilon) C_{\theta} \widetilde{S}_{\theta} \rho_{0}+\varepsilon\left(C_{\theta} \widetilde{S}_{\theta}+S_{\theta} \widetilde{C}_{\theta}\right) \frac{\sigma}{\sigma^{\prime}}\right)\left(\left(C_{\theta} \widetilde{S}_{\theta}-\varepsilon \widetilde{\varepsilon} S_{\theta} \widetilde{C}_{\theta}\right)+\varepsilon \widetilde{\varepsilon} S_{\theta} \widetilde{C}_{\theta} \zeta_{0}\right)\right] \\
& -a\left[\left(\left(C_{\theta} \widetilde{C}_{\theta}-\varepsilon S_{\theta} \widetilde{S}_{\theta}\right)+\varepsilon S_{\theta} \widetilde{S}_{\theta} \zeta_{0}\right)\left((1-\varepsilon \widetilde{\varepsilon}) C_{\theta} \widetilde{C}_{\theta} \rho_{0}+\left(\varepsilon \widetilde{\varepsilon} C_{\theta} \widetilde{C}_{\theta}+\varepsilon S_{\theta} \widetilde{S}_{\theta}\right) \frac{\sigma}{\sigma^{\prime}}\right)\right]
\end{aligned}
$$




$$
\begin{aligned}
& =a\left[(\varepsilon \widetilde{\varepsilon}-1) C_{\theta}^{2}\left(\widetilde{C}_{\theta}^{2}-\widetilde{\varepsilon} \widetilde{S}_{\theta}^{2}\right) \rho_{0}+\left(S_{\theta}^{2}+\varepsilon C_{\theta}^{2}\right)\left(\widetilde{S}_{\theta}^{2}-\widetilde{\varepsilon} \widetilde{C}_{\theta}^{2}\right) \frac{\sigma}{\sigma^{\prime}}\right. \\
& \left.+\widetilde{\varepsilon} S_{\theta}^{2}\left(\widetilde{C}_{\theta}^{2}-\widetilde{\varepsilon} \widetilde{S}_{\theta}^{2}\right) \frac{\sigma}{\sigma^{\prime}} \zeta_{0}\right]
\end{aligned}
$$

and due to the identity $(59)$ and the hypothesis $(66)$, we have the intermediate result :

$$
\Delta=a\left[(\varepsilon \widetilde{\varepsilon}-1) C_{\theta}^{2} \rho_{0}-\widetilde{\varepsilon}\left(S_{\theta}^{2}+\varepsilon C_{\theta}^{2}\right) \frac{\sigma}{\sigma^{\prime}}+\widetilde{\varepsilon} S_{\theta}^{2} \frac{\sigma}{\sigma^{\prime}} \zeta_{0}\right] .
$$

Then

$$
\begin{aligned}
\Delta & =a \frac{\sigma}{\sigma^{\prime}}\left[(\varepsilon \widetilde{\varepsilon}-1) C_{\theta}^{2}\left(\frac{\sigma^{*}}{\sigma}+1\right)-\widetilde{\varepsilon}\left(S_{\theta}^{2}+\varepsilon C_{\theta}^{2}\right)+\widetilde{\varepsilon} S_{\theta}^{2} \zeta_{0}\right] \\
& =a \frac{\sigma}{\sigma^{\prime}}\left[(\varepsilon \widetilde{\varepsilon}-1) C_{\theta}^{2} \frac{\sigma^{*}}{\sigma}-\left(C_{\theta}^{2}+\widetilde{\varepsilon} S_{\theta}^{2}\right)+\widetilde{\varepsilon} S_{\theta}^{2} \zeta_{0}\right]
\end{aligned}
$$

and the expression $(69)$ is established.

- After determining the parameters $\theta$ and $\rho_{0}$ it is possible to evaluate the mathematical entropy $\eta$ thanks to the relation (31). Then we can differentiate this mathematical entropy relatively the density $\rho$ and the momentum $J$. We have the following remarquable coherence property.

\section{- Proposition 14. Entropy variables}

If the jacobian determinant 69 is not null, then the entropy variables $\varphi \equiv(\alpha, \beta)$ can be determined according to the relations 500 . In other terms,

$$
\alpha \equiv \frac{\partial \eta}{\partial \rho}=\widetilde{C}_{\theta} \sigma^{\prime}\left(\rho_{0}\right), \quad \beta \equiv \frac{\partial \eta}{\partial J}=-\frac{\widetilde{\varepsilon}}{a} \widetilde{S}_{\theta} \sigma^{\prime}\left(\rho_{0}\right)
$$

\section{Proof of Proposition 14.}

We first invert the jacobian matrix :

Then

$$
\left(\begin{array}{ll}
\frac{\partial \rho}{\partial \theta} & \frac{\partial \rho}{\partial \rho_{0}} \\
\frac{\partial J}{\partial \theta} & \frac{\partial J}{\partial \rho_{0}}
\end{array}\right)^{-1}=\left(\begin{array}{ll}
\frac{\partial \theta}{\partial \rho} & \frac{\partial \theta}{\partial J} \\
\frac{\partial \rho_{0}}{\partial \rho} & \frac{\partial \rho_{0}}{\partial J}
\end{array}\right)=\frac{1}{\Delta}\left(\begin{array}{cc}
\frac{\partial J}{\partial \rho_{0}} & -\frac{\partial \rho}{\partial \rho_{0}} \\
-\frac{\partial J}{\partial \theta} & \frac{\partial \rho}{\partial \theta}
\end{array}\right)
$$

$$
\left\{\begin{aligned}
\frac{\partial \theta}{\partial \rho} & =\frac{1}{\Delta} \frac{\partial J}{\partial \rho_{0}}=\frac{a}{\Delta}\left[\left(C_{\theta} \widetilde{S}_{\theta}-\varepsilon \widetilde{\varepsilon} S_{\theta} \widetilde{C}_{\theta}\right)+\varepsilon \widetilde{\varepsilon} S_{\theta} \widetilde{C}_{\theta} \zeta_{0}\right] \\
\frac{\partial \rho_{0}}{\partial \rho} & =-\frac{1}{\Delta} \frac{\partial J}{\partial \theta}=-\frac{a}{\Delta}\left[(1-\varepsilon \widetilde{\varepsilon}) C_{\theta} \widetilde{C}_{\theta} \rho_{0}+\left(\varepsilon \widetilde{\varepsilon} C_{\theta} \widetilde{C}_{\theta}+\varepsilon S_{\theta} \widetilde{S}_{\theta}\right) \frac{\sigma}{\sigma^{\prime}}\right] \\
\frac{\partial \theta}{\partial J} & =-\frac{1}{\Delta} \frac{\partial \rho}{\partial \rho_{0}}=-\frac{1}{\Delta}\left[\left(C_{\theta} \widetilde{C}_{\theta}-\varepsilon S_{\theta} \widetilde{S}_{\theta}\right)+\varepsilon S_{\theta} \widetilde{S}_{\theta} \zeta_{0}\right] \\
\frac{\partial \rho_{0}}{\partial J}=\frac{1}{\Delta} \frac{\partial \rho}{\partial \theta} & =\frac{1}{\Delta}\left[(\widetilde{\varepsilon}-\varepsilon) C_{\theta} \widetilde{S}_{\theta} \rho_{0}+\varepsilon\left(C_{\theta} \widetilde{S}_{\theta}+S_{\theta} \widetilde{C}_{\theta}\right) \frac{\sigma}{\sigma^{\prime}}\right] .
\end{aligned}\right.
$$

We first observe that

$$
\frac{\partial \eta}{\partial \theta}=\frac{\partial}{\partial \theta}\left(C_{\theta} \sigma\left(\rho_{0}\right)\right)=\varepsilon S_{\theta} \sigma, \quad \frac{\partial \eta}{\partial \rho_{0}}=\frac{\partial}{\partial \rho_{0}}\left(C_{\theta} \sigma\left(\rho_{0}\right)\right)=C_{\theta} \sigma^{\prime}
$$

Then with the chain rule, we have

$$
\alpha=\frac{\partial \eta}{\partial \rho}=\frac{\partial \eta}{\partial \theta} \frac{\partial \theta}{\partial \rho}+\frac{\partial \eta}{\partial \rho_{0}} \frac{\partial \rho_{0}}{\partial \rho}
$$




$$
\begin{aligned}
=\frac{a}{\Delta}\left[\varepsilon S_{\theta} \sigma\left(\left(C_{\theta} \widetilde{S}_{\theta}-\varepsilon \widetilde{\varepsilon} S_{\theta} \widetilde{C}_{\theta}\right)+\varepsilon \widetilde{\varepsilon} S_{\theta} \widetilde{C}_{\theta} \zeta_{0}\right)\right. & \\
& \quad-C_{\theta} \sigma^{\prime}\left((1-\varepsilon \widetilde{\varepsilon}) C_{\theta} \widetilde{C}_{\theta} \rho_{0}+\left(\varepsilon \widetilde{\varepsilon} C_{\theta} \widetilde{C}_{\theta}+\varepsilon S_{\theta} \widetilde{S}_{\theta} \frac{\sigma}{\sigma^{\prime}}\right)\right] \\
= & \frac{a}{\Delta}\left[(\varepsilon \widetilde{\varepsilon}-1) C_{\theta}^{2} \widetilde{C}_{\theta} \rho_{0} \sigma^{\prime}+\widetilde{\varepsilon} S_{\theta}^{2} \widetilde{C}_{\theta} \zeta_{0} \sigma-\varepsilon \widetilde{C}_{\theta}\left(S_{\theta}^{2}+\varepsilon C_{\theta}^{2}\right) \sigma\right] \\
= & \frac{a}{\Delta} \widetilde{C}_{\theta} \sigma^{\prime}\left[(\varepsilon \widetilde{\varepsilon}-1) C_{\theta}^{2} \rho_{0}+\widetilde{\varepsilon} S_{\theta}^{2} \zeta_{0} \frac{\sigma}{\sigma^{\prime}}-\varepsilon\left(S_{\theta}^{2}+\varepsilon C_{\theta}^{2}\right) \frac{\sigma}{\sigma^{\prime}}\right]=\widetilde{C}_{\theta} \sigma^{\prime}
\end{aligned}
$$

due to $(70)$. In an analogous way, we have

$$
\begin{aligned}
& \beta= \frac{\partial \eta}{\partial J}=\frac{\partial \eta}{\partial \theta} \frac{\partial \theta}{\partial J}+\frac{\partial \eta}{\partial \rho_{0}} \frac{\partial \rho_{0}}{\partial J} \\
&=\frac{1}{\Delta}[ \quad-\varepsilon S_{\theta} \sigma\left(\left(C_{\theta} \widetilde{C}_{\theta}-\varepsilon S_{\theta} \widetilde{S}_{\theta}\right)+\varepsilon S_{\theta} \widetilde{S}_{\theta} \zeta_{0}\right) \\
&\left.\quad+C_{\theta} \sigma^{\prime}\left((\widetilde{\varepsilon}-\varepsilon) C_{\theta} \widetilde{S}_{\theta} \rho_{0}+\varepsilon\left(C_{\theta} \widetilde{S}_{\theta}+S_{\theta} \widetilde{C}_{\theta}\right) \frac{\sigma}{\sigma^{\prime}}\right)\right] \\
&=\frac{\widetilde{\varepsilon}}{\Delta}\left[(1-\varepsilon \widetilde{\varepsilon}) C_{\theta}^{2} \widetilde{S}_{\theta} \rho_{0} \sigma^{\prime}+\widetilde{\varepsilon}\left(S_{\theta}^{2}+\varepsilon C_{\theta}^{2}\right) \widetilde{S}_{\theta} \sigma-\widetilde{\varepsilon} S_{\theta}^{2} \widetilde{S}_{\theta} \zeta_{0} \sigma\right] \\
&=\frac{\widetilde{\varepsilon}}{\Delta} \widetilde{S}_{\theta} \sigma^{\prime}\left[(1-\varepsilon \widetilde{\varepsilon}) C_{\theta}^{2} \rho_{0}+\widetilde{\varepsilon}\left(S_{\theta}^{2}+\varepsilon C_{\theta}^{2}\right) \frac{\sigma}{\sigma^{\prime}}-\widetilde{\varepsilon} S_{\theta}^{2} \zeta_{0} \frac{\sigma}{\sigma^{\prime}}\right]=-\frac{\widetilde{\varepsilon}}{a} \widetilde{S}_{\theta} \sigma^{\prime}
\end{aligned}
$$

due to 70 . The proof is completed.

- We particularize our study and wish to construct nontrivial hyperbolic systems with entropy velocity covariant under the Lorentz group and - or the circular group. It seems essential to maintain a constant sign for the determinant $\Delta$ presented at the relation (69). Moreover, we have to enforce the strict convexity of the mathematical entropy $(\rho, J) \longmapsto \eta(\rho, J)$. In this contribution, we only consider the particular case

$$
\varepsilon=\widetilde{\varepsilon}
$$

We focus on "circular elliptic" and "Lorentz hyperbolic" two by two systems of conservation laws. Observe first that due to the condition (72), the expression (67) of the conserved variables simplify into

$$
\rho=\rho_{0}+\varepsilon S_{\theta}^{2} \frac{\sigma}{\sigma^{\prime}}, \quad J=a S_{\theta} C_{\theta} \frac{\sigma}{\sigma^{\prime}} .
$$

Then the mathematical entropy $\eta$ constrained to the condition (31) is strictly convex if and only if the determinant of its hessian $\operatorname{det}\left(\mathrm{d}^{2} \eta\right)$ is stricly positive and if the second derivative $\frac{\partial^{2} \eta}{\partial \rho^{2}}$ is also strictly positive.

\section{- Proposition 15. Technical lemma for convexity conditions}

Under the hypotheses (66) and (72), the expression of the jacobian determinant $\Delta$ is given according to

$$
\Delta=a \delta \frac{\sigma}{\sigma^{\prime}}
$$

with

$$
\delta=\varepsilon S_{\theta}^{2} \zeta_{0}-\left(C_{\theta}^{2}+\varepsilon S_{\theta}^{2}\right) .
$$

The two signed expressions are given according to

$$
\operatorname{det}\left(\mathrm{d}^{2} \eta\right)=\frac{\varepsilon \sigma^{\prime} \sigma^{\prime \prime}}{a \Delta}=\frac{\varepsilon}{a^{2}}\left(\sigma^{\prime}\right)^{2} \frac{\sigma^{\prime \prime}}{\sigma} \frac{1}{\delta}
$$


and

$$
\frac{\partial^{2} \eta}{\partial \rho^{2}}=\frac{\partial \alpha}{\partial \rho}=\frac{C_{\theta}}{\delta}\left(\varepsilon S_{\theta}^{2} \zeta_{0} \frac{\sigma^{\prime 2}}{\sigma}-\left(C_{\theta}^{2}+\varepsilon S_{\theta}^{2}\right) \sigma^{\prime \prime}\right) .
$$

\section{Proof of Proposition 15.}

The expression $\delta$ in $(75)$ is just a specific name for the big braket in right hand side of the expression (69) and the relation (74) is satisfied. Under the condition (72), the expression $\delta$ is clearly reduced to the expression (75). We establish now the expression $(76)$ of the determinant of the hessian matrix. From the chain rule, we have

$$
\begin{aligned}
\operatorname{det}\left(\mathrm{d}^{2} \eta\right) & =\frac{\partial(\alpha, \beta)}{\partial(\rho, J)}=\frac{\partial(\alpha, \beta)}{\partial\left(\theta, \rho_{0}\right)} \frac{\partial\left(\theta, \rho_{0}\right)}{\partial(\rho, J)}=\frac{1}{\Delta} \operatorname{det}\left(\begin{array}{cc}
\frac{\partial \alpha}{\partial \theta} & \frac{\partial \alpha}{\partial \rho_{0}} \\
\frac{\partial \beta}{\partial \theta} & \frac{\partial \beta}{\partial \rho_{0}}
\end{array}\right) \\
& =\frac{1}{\Delta} \operatorname{det}\left(\begin{array}{cc}
\varepsilon S_{\theta} \sigma^{\prime} & C_{\theta} \sigma^{\prime \prime} \\
-\frac{\varepsilon}{a} C_{\theta} \sigma^{\prime} & -\frac{\varepsilon}{a} S_{\theta} \sigma^{\prime \prime}
\end{array}\right)=-\frac{1}{\Delta} \frac{\varepsilon}{a} \sigma^{\prime} \sigma^{\prime \prime} \operatorname{det}\left(\begin{array}{cc}
\varepsilon S_{\theta} & C_{\theta} \\
C_{\theta} & S_{\theta}
\end{array}\right)
\end{aligned}
$$

due to 71 and 72 . Then $\operatorname{det}\left(\mathrm{d}^{2} \eta\right)=\frac{1}{a \delta} \frac{\sigma^{\prime}}{\sigma} \frac{\varepsilon}{a} \sigma^{\prime} \sigma^{\prime \prime}$ is a consequence of the trigonometrical property (16) and the expression (76) follows. The evaluation of (77) is also elementary. From the chain rule, we have

$$
\begin{aligned}
\frac{\partial \alpha}{\partial \rho} & =\frac{\partial \alpha}{\partial \theta} \frac{\partial \theta}{\partial \rho}+\frac{\partial \alpha}{\partial \rho_{0}} \frac{\partial \rho_{0}}{\partial \rho}=\frac{a}{\Delta}\left[\varepsilon S_{\theta} \sigma^{\prime} S_{\theta} C_{\theta} \zeta_{0}-C_{\theta} \sigma^{\prime \prime}\left(C_{\theta}^{2}+\varepsilon S_{\theta}^{2}\right) \frac{\sigma}{\sigma^{\prime}}\right] \\
& =\frac{1}{\delta} \frac{\sigma^{\prime}}{\sigma} C_{\theta}\left[\varepsilon S_{\theta}^{2} \sigma^{\prime} \zeta_{0}-\sigma^{\prime \prime}\left(C_{\theta}^{2}+\varepsilon S_{\theta}^{2}\right) \frac{\sigma}{\sigma^{\prime}}\right]
\end{aligned}
$$

and the expression (77) is clear.

- We detail now two explicit examples of hyperbolic system of order two. The first one is invariant under the action of the circular group and the second under the Lorentz group.

\section{- Proposition 16. Exponential entropy for a "circular-elliptic" system}

We introduce two strictly positive constants $\rho_{*}$ and $\bar{\sigma}$ and an exponential entropy on the manifold $\Omega_{0}$ thanks to

$$
\sigma\left(\rho_{0}\right)=\bar{\sigma} \exp \left(\frac{\rho_{0}}{\rho_{*}}\right)
$$

We set

$$
\begin{cases}\Psi=\frac{2 J}{a \rho_{*}}, \quad \rho_{0}=\rho+\frac{1}{2}\left(1-\sqrt{1-\Psi^{2}}\right) \rho_{*}, & \theta=\frac{1}{2} \operatorname{Arcsin} \Psi, \\ u=c \frac{J}{|J|} \sqrt{\frac{1-\sqrt{1-\Psi^{2}}}{1+\sqrt{1-\Psi^{2}}}}=c \operatorname{sgn}(J) \tan \theta, & p_{0}=a c\left(\rho_{0}-\rho_{*}\right) .\end{cases}
$$

The system of conservation laws

$$
\left\{\begin{aligned}
\frac{\partial \rho}{\partial t}+\frac{\partial}{\partial x}\left(\frac{c}{a} J\right) & =0 \\
\frac{\partial J}{\partial t}+\frac{\partial}{\partial x}\left(u J+p_{0}\right) & =0
\end{aligned}\right.
$$




\section{François Dubois}

is hyperbolic if we suppose

$$
|\Psi| \leq 1, \quad|\theta| \leq \frac{\pi}{4} .
$$

It admits a mathematical entropy given by the expression

$$
\eta(\rho, J) \equiv \sqrt{\frac{1}{2}\left(1+\sqrt{1-\Psi^{2}}\right)} \sigma\left(\rho+\frac{\rho_{*}}{2}\left(1-\sqrt{1-\Psi^{2}}\right)\right)
$$

and the dissipation of entropy of entropy solutions is given by the inequality

$$
\frac{\partial \eta}{\partial t}+\frac{\partial}{\partial x}(u \eta) \leq 0 .
$$

\section{Proof of Proposition 16.}

With the choice $(78)$ of an exponential entropy, the parameter $\zeta_{0}$ introduced at the relation (68) is null. Because $\bar{\sigma}$ is supposed strictly positive, $\sigma$ is a convex function and the condition $\operatorname{det}\left(\mathrm{d}^{2} \eta\right)>0$ is reduced to $\delta<0$ if we look to a "circular-elliptic" hyperbolic system, id est if we suppose $\varepsilon=-1$ (see (76)). Then with the notation $\Psi$ introduced in (79), the relations (73) can be written

$$
\sin (2 \theta)=\Psi, \quad \rho=\rho_{0}-\sin ^{2} \theta \rho_{*} .
$$

and the calculus of $\theta$ proposed in 790 is natural. We have the same remark for the expression of $\rho_{0}$ in 790 as a consequence of the identity $\sin ^{2} \theta=\frac{1}{2}\left(1-\sqrt{1-\sin ^{2}(2 \theta)}\right)$. The second condition in (81) is a consequence of the expression of $\theta$ proposed in (79). This last condition is also necessary when we impose $\delta<0$ because the relation (75) can be now written $\delta=-\left(\cos ^{2} \theta-\sin ^{2} \theta\right)$. The velocity in 79 is simply the actual formulation of the relation 30 because

$$
\tan \theta=\operatorname{sgn}(\theta) \sqrt{\frac{1-\sqrt{1-\sin ^{2}(2 \theta)}}{1+\sqrt{1+\sin ^{2}(2 \theta)}}} .
$$

- The static pressure $p_{0}$ is related to the entropy $\sigma$ thanks to (63). The expressions (8) and (33) of the physical and thermodynamix fluxes, the algebraic forms (58) of the matrix $Y_{\theta}$ and the choice (62) for $g_{0}$ show that the first component $f_{1}$ of $f(W)$ is given by

$$
\begin{aligned}
f_{1} & =\rho u-\frac{\sin \theta}{a \cos \theta} p_{0}=\rho u-\frac{1}{a c} u a c\left(\rho_{0}-\rho_{*}\right)=\rho_{*} u\left(1-\sin ^{2} \theta\right) \\
& =\rho_{*} c \sin \theta \cos \theta=\frac{1}{2} \rho_{*} c \sin (2 \theta)=\frac{1}{2} \rho_{*} c \Psi=\frac{1}{2} \rho_{*} c \frac{2 J}{a \rho_{*}}=\frac{c}{a} J .
\end{aligned}
$$

Then the relations 80 are established. The expression 82 of the mathematical entropy is then a consequence of (31) : $\eta=\cos \theta \sigma\left(\rho_{0}\right)$. A particular hyperbolic system with entropy velocity invariant under the action of the circular group is constructed. 
- Proposition 17. Homographic entropy for a "Lorentz-hyperbolic" system We consider two strictly positive constants $\rho_{*}$ and $\bar{\sigma}$ and an homographic entropy $\sigma$ on the manifold $\Omega_{0}$ defined by the relation

$$
\sigma\left(\rho_{0}\right)=-\bar{\sigma} \frac{\rho_{0}}{\rho_{0}+\rho_{*}}, \quad \rho_{0}>0 .
$$

We set

$$
\Phi\left(\rho_{0}\right) \equiv \frac{2 J}{a} \frac{\sigma^{\prime}}{\sigma}=\frac{2 J}{a} \frac{\rho_{*}}{\rho_{0}\left(\rho_{0}+\rho_{*}\right)}
$$

and we suppose

$$
\frac{|J|}{a} \leq \rho \leq \frac{1}{2} \rho_{*}
$$

Then the equation

$$
F\left(\rho_{0}\right) \equiv \rho_{0}+\frac{\sigma}{2 \sigma^{\prime}}\left(\sqrt{1+\Phi\left(\rho_{0}\right)^{2}}-1\right)=\rho
$$

has a unique solution $\rho_{0}$ such that

$$
0 \leq \rho_{0} \leq \frac{1}{2} \rho_{*}
$$

We consider the expressions

$$
p_{0}=a c \frac{\rho_{0}^{2}}{\rho_{*}}, \quad \theta=\frac{1}{2} \operatorname{Argsh} \Phi\left(\rho_{0}\right), \quad u=a \tanh \theta=c \operatorname{sgn}(J) \sqrt{\frac{\sqrt{1+\Phi^{2}}-1}{\sqrt{1+\Phi^{2}}+1}} .
$$

Then the system of conservation laws

$$
\left\{\begin{aligned}
\frac{\partial \rho}{\partial t}+\frac{\partial}{\partial x}\left[\left(\rho+\frac{\rho_{0}}{\rho_{*}}\right) u\right] & =0 \\
\frac{\partial J}{\partial t}+\frac{\partial}{\partial x}\left(u J+p_{0}\right) & =0
\end{aligned}\right.
$$

is hyperbolic and admits a mathematical entropy given by the expression

$$
\eta(\rho, J) \equiv \sqrt{\frac{1}{2}\left(1+\sqrt{1+\Phi(\rho)^{2}}\right)} \sigma\left(\rho_{0}\right) .
$$

The dissipation of entropy of entropy solutions is still given by the inequality 83 .

\section{Proof of Proposition 17.}

In this case of the Lorentz group, we have $\varepsilon=1, S_{\theta} \equiv \sinh \theta$ and $C_{\theta} \equiv \cosh \theta$. We have chosen with (84) a negative convex function $\sigma$. Then (c.f. (76) ) the condition $\delta<0$ has to be satisfied to assume $\operatorname{det}\left(\mathrm{d}^{2} \eta\right)>0$. We have

The relations $(73)$ can now be written as

$$
\frac{\sigma}{\sigma^{\prime}}=\frac{\rho_{0}}{\rho_{*}}\left(\rho_{0}+\rho_{*}\right), \quad \zeta_{0} \equiv \frac{\partial}{\partial \rho_{0}}\left(\frac{\sigma}{\sigma^{\prime}}\right)=1+2 \frac{\rho_{0}}{\rho_{*}} .
$$

$$
\sinh (2 \theta)=\Phi\left(\rho_{0}\right), \quad \rho=\rho_{0}+\sinh ^{2} \theta \frac{\sigma}{\sigma^{\prime}} .
$$

The identity $\sinh ^{2} \theta \equiv \frac{1}{2}\left(\sqrt{1+\sinh ^{2}(2 \theta)}-1\right)$ shows that the density $\rho_{0}$ at null velocity associated with an arbitrary state $W \equiv(\rho, J)^{\mathrm{t}}$ has to solve the equation

$$
\rho-\rho_{0}=\frac{1}{2}\left(\sqrt{1+\Phi\left(\rho_{0}\right)^{2}}-1\right) \frac{\sigma}{\sigma^{\prime}}
$$




\section{FrançOIS Dubois}

which is equivalent to (87). We remark that $F(0)=|J| / a$ and the first condition of (88) is natural.

- We prove now that the equation (87) has a unique solution if the conditions (86) are satisfied. We have

$$
\frac{\partial}{\partial \rho_{0}}\left[\frac{\sigma}{2 \sigma^{\prime}} \sqrt{1+\Phi^{2}}\right]=\frac{1}{2} \frac{\partial}{\partial \rho_{0}} \sqrt{\left(\frac{\sigma}{\sigma^{\prime}}\right)^{2}+\frac{4 J^{2}}{a^{2}}}=\frac{2}{4 \sqrt{\left(\frac{\sigma}{\sigma^{\prime}}\right)^{2}+\frac{4 J^{2}}{a^{2}}}} \frac{\sigma}{\sigma^{\prime}} \zeta_{0}>0
$$

if $\rho_{0}>0$. We have also $\frac{\partial}{\partial \rho_{0}}\left(\rho_{0}-\frac{\sigma}{2 \sigma^{\prime}}\right)=1-\frac{\zeta_{0}}{2}=\frac{1}{2}-\frac{\rho_{0}}{\rho_{*}}>0 \quad$ under the condition (88) for $\rho_{0}$. Then we have $F^{\prime}\left(\rho_{0}\right)>0$ and the equation (87) has a unique solution when 88 is satisfied. The maximum of the function $F(\bullet)$ on the interval $\left[0, \frac{1}{2} \rho_{*}\right]$ can be estimated as follows. We have first $\frac{\sigma}{\sigma^{\prime}}\left(\frac{\rho_{*}}{2}\right)=\frac{1}{2}\left(\frac{1}{2}+1\right) \rho_{*}=\frac{3}{4} \rho_{*}$. Then

$$
F\left(\frac{\rho_{*}}{2}\right)=\frac{\rho_{*}}{2}-\frac{3}{8} \rho_{*}+\frac{\rho_{*}}{2} \sqrt{\left(\frac{3}{4}\right)^{2}+\left(\frac{2 J}{a \rho_{*}}\right)^{2}} \geq \frac{\rho_{*}}{2}-\frac{3}{8} \rho_{*}+\frac{3}{8} \rho_{*}=\frac{\rho_{*}}{2}
$$

and the second condition of $(86)$ is natural. The pressure $p_{0}$ is given by the relation (63) :

$$
p_{0}=-a c \frac{\sigma^{*}}{\sigma^{\prime}}=a c\left(\frac{\sigma}{\sigma^{\prime}}-\rho_{0}\right)=a c \frac{\rho_{0}^{2}}{\rho_{*}}
$$

and the first relation of $(89)$ is established. The two other relations of $(89)$ are an elementary consequence of the previous considerations.

- We now establish the algebraic form of the hyperbolic system (1). From (8) and (33), the particular expression 58 of the matrix $Y_{\theta}$ and the choice $g_{0} \equiv\left(0, p_{0}\right)^{\mathrm{t}}$ proposed in (62) show that the first component $f_{1}$ of $f(W)$ is given by

$$
f_{1}=\rho u+\frac{\sinh \theta}{a \cosh \theta} p_{0}=\rho u+\frac{1}{a c} u a c \frac{\rho_{0}^{2}}{\rho_{*}}=\left(\rho+\frac{\rho_{0}^{2}}{\rho_{*}}\right) u .
$$

Then the fist line of the relations $(90)$ is established. The second line is straightforword to evidence. The expression of the mathematical entropy is a direct consequence of (31) and the explitation of $\cosh \theta$ as a function of $\Phi\left(\rho_{0}\right)$ as proposed in (89). This remark completes the construction of an hyperbolic system with entropy velocity $(90)$ invariant under the action of the Lorentz group.

\section{7) Conclusion}

- In this contribution, we have extended our previous work [6] relative to the construction of hyperbolic systems covariant under the Galileo group and space reflection. We have precised the notion of "entropy velocity" as an intrinsic velocity associated with the conservation of entropy for regular solutions. In particular, the usual velocity of gas dynamics system is an entropy velocity. An hyperbolic system of conservation laws and the associated conservation of entropy are covariant under the action of a group of space-time transformations if the algebraic form of the equations is not changed after the action of the corresponding group. We have proposed sufficient conditions in order to satisfy a group covariance property. These conditions introduce naturally a representation of the 
group in the space of states that can be chosen in multiple ways. We have also introduced a natural manifold of "null velocity". When thermodynamic data are given on this manifold, the natural question is the construction of the whole hyperbolic system from the covariance property. We have also natural constraints for the entropy variables.

- After the study of the particular case of Galileo covariance in [6], we have particularized in this contribution our study for the two-dimensional case with the Lorentz and circular groups. We have proposed nontrivial hyperbolic systems covariant under each of these two groups. For the circular example, we find a condition (81) that shows that the associated hyperbolic system is not covariant under the action of the whole circular group. The question is set [23] to know if there exists a mathematical obstruction to find hyperbolic systems covariant under the action of the whole circular group. A better knowledge of such systems of conservation laws is a possible next step. Last but not least, the link with relativistic gas dynamics (see e.g. Souriau [20, 21], Chiu [3] and Vallée [22]) is also a natural question. The extension of this work to several space dimensions is, in principle, straightforward.

\section{Acknowledgments}

The author thanks Géry de Saxcé and Claude Vallée for their kind invitation to participate to the 56th and 57th CITV in august 2012 and august 2013, and for stimulating discussions during these workshops. In november 2014, Claude Vallée passed away. Of course, this contribution is also dedicated to him.

\section{References}

[1] V. Bargmann. "On unitary ray representations of continuous groups", Annals of Mathematics, vol. 59, p. 1-46, 1954.

[2] G. Boillat. "Sur l'existence et la recherche d'équations de conservation supplémentaires pour les systèmes hyperboliques", Comptes Rendus de l'Académie des Sciences, série A, tome 278, p. 909-912, 1974.

[3] H.H. Chiu. "Relativistic gas dynamics", Physics of Fluids, vol. 16, p. 825-830, 1973.

[4] G. de Saxcé, C. Vallée. "Bargmann group, momentum tensor and Galilean invariance of Clausius-Duhem inequality", International Journal of Engineering Science, vol. 50, p. 216-232, 2012.

[5] B. Després, F. Dubois. Systèmes hyperboliques de lois de conservation ; application à la dynamique des gaz, Editions de l'Ecole Polytechnique, Palaiseau, 2005.

[6] F. Dubois. "Conservation laws invariants for Galileo group; CEMRACS preliminary results", ESAIM: Proc., vol. 10, p. 233 - 266, 2001.

[7] W. Fenchel. "On conjugate convex functions", Canadian Journal of Mathematics, vol. 1, p. 73-77, 1949. 


\section{FranÇOIS DUBOIS}

[8] K.O. Friedrichs, P.D. Lax. "Systems of Conservation Equations with a Convex Extension", Proceedings of the National Academy of Sciences of the USA, vol. 68, p. 16861688, 1971.

[9] E. Godlewski, P.A. Raviart. Numerical Approximation of Hyperbolic Systems of Conservation Laws, Springer-Verlag, New York Inc., 1996.

[10] S. K. Godunov. "An interesting class of quasilinear systems", Dokl. Akad. Nauk SSSR, vol. 139, p. 521-523 ; see also Soviet. Math., vol. 2, p. 947-949, 1961.

[11] B. Kostant. "Quantization and Unitary Representations", Lecture Notes in Mathematics, Springer-Verlag, vol. 170, p. 87-207, 1970.

[12] S. Kružkov. "First Order Quasi-Linear Systems in Several Independant Variables", Math. Sbornik, vol. 123, p. 228-255, and Math. USSR Sb., vol. 10, no 2, p. 217-243, 1970.

[13] P.D. Lax. Hyperbolic Partial Differential Equations, American Mathematical Society, Courant Lecture Notes, vol. 14, 2006.

[14] A.M. Legendre. "Mémoire sur l'intégration de quelques équations aux différences partielles", Mémoire de l'Académie Royale des Sciences, année 1787, p. 309 - 351, 1787.

[15] J.J. Moreau. "Fonctions convexes duales et points proximaux dans un espace hilbertien", Comptes Rendus de l'Académie des Sciences, série A, tome 255, p. 2897-2899, 1962.

[16] E. Noether. "Invariante Variationsprobleme", Nachrichten von der Königlicher Gesellschaft den Wissenschaft zu Göttingen, Mathematisch-Physikalische Klasse, p. 235-257, 1918.

[17] T. Ruggeri. "Galilean invariance and entropy principle for systems of balance laws; the structure of extended thermodynamics", Continuum Mechanics and Thermodynamics, vol. 1, p. 3-20, 1989.

[18] J.M. Souriau. Géométrie et relativité, Hermann, Paris, 1964.

[19] J.M. Souriau. Structure des systèmes dynamiques, Dunod, Paris, 1970.

[20] J.M. Souriau. "Thermodynamique relativiste des fluides", Prépublication CPT77/P.919, Centre de Physique Théorique, Marseille, mai 1997.

[21] J.M. Souriau. "Thermodynamique et géométrie", in Differential geometrical methods in mathematical physics II, Proceedings, University of Bonn, July 13-16, 1977, Lecture Notes in Math., vol. 676, Bleuler et al. Editors, p. 369-397, Springer, Berlin, 1978.

[22] C. Vallée. "Lois de comportement des milieux continus dissipatifs compatibles avec la physique relativiste", thèse, Poitiers University, 1987.

[23] C. Vallée. Personal communication, CITV, La Baume, Aix en Provence, 22 August 2013. 